LAWRENCE LIVERMORE N A T IO N A L LABORATORY

\title{
Realizing the Opportunities of Neutron Cross Section Measurements at RIA
}

L. Ahle, M. Hausmann, R. Reifarth, K. Roberts, M. Roeben, B. Rusnak, D. Vieira

October 19, 2004

International Conference on Nuclear Data for Science and Technology Santa Fe, NM, United States September 26, 2004 through October 1, 2004 
This document was prepared as an account of work sponsored by an agency of the United States Government. Neither the United States Government nor the University of California nor any of their employees, makes any warranty, express or implied, or assumes any legal liability or responsibility for the accuracy, completeness, or usefulness of any information, apparatus, product, or process disclosed, or represents that its use would not infringe privately owned rights. Reference herein to any specific commercial product, process, or service by trade name, trademark, manufacturer, or otherwise, does not necessarily constitute or imply its endorsement, recommendation, or favoring by the United States Government or the University of California. The views and opinions of authors expressed herein do not necessarily state or reflect those of the United States Government or the University of California, and shall not be used for advertising or product endorsement purposes. 


\title{
Realizing the Opportunities of Neutron Cross Section Measurements at RIA
}

\author{
Larry Ahle*, Marc Hausmann ${ }^{+}$, Rene Reifarth ${ }^{+}$, Kevin Roberts*, Martin Roeben*, \\ Brian Rusnak*, Dave Vieira ${ }^{+}$ \\ *Lawrence Livermore National Laboratory, Livermore, CA 94550, USA \\ ${ }^{+}$Los Alamos National Laboratory, Los Alamos, NM 87545, USA
}

\begin{abstract}
The Rare Isotope Accelerator will produce many isotopes at never before seen rates. This will allow for the first time measurements on isotopes very far from stability and new measurement opportunities for unstable nuclei near stability. In fact, the production rates are such that it should be possible to collect 10 micrograms of many isotopes with a half-life of 1 day or more. This ability to make targets of short-lived nuclei enables the possibility of making neutron cross-section measurements important to the astrophysics and the stockpile stewardship communities. But to fully realize this opportunity, the appropriate infrastructure must be included at the RIA facility. This includes isotope harvesting capabilities, radiochemical areas for processing collected material, and an intense, "mono-energetic", tunable neutron source. As such, we have been developing a design for neutron source facility to be included at the RIA site. This facility would produce neutrons via intense beams of deuterons and protons on a variety of targets. The facility would also include the necessary radiochemical facilities for target processing. These infrastructure needs will be discussed in addition to the methods that would be employed at RIA for measuring these neutron cross-sections.
\end{abstract}

\section{INTRODUCTION}

An intense neutron source facility with radiochemical processing capability is necessary at the Rare Isotope Accelerator to fully realize its potential benefit to stockpile stewardship and astrophysics. While many of the important physics missions of RIA can be addressed with radioactive ion beams, direct neutron cross-section measurements of interest to stockpile stewardship and astrophysics cannot because of the impossibility of a neutron target. The unprecedented production rates expected at RIA enables many of these direct neutron cross-section measurements, but only if the proper infrastructure is in place.

\section{MOTIVATION FOR NEUTRON CROSS SECTION MEASURMENTS}

To understand nuclear weapon test data and the origin of the elements heavier than iron, a detailed knowledge of neutron cross-section on a number of near-stability nuclei is required. For astrophysics, $(\mathrm{n}, \gamma)$ cross-sections on neutron-rich nuclei are required, while for stockpile stewardship $(\mathrm{n}, \gamma)$ and $(n, 2 n)$ cross-sections for proton-rich nuclei are the most important neutron reactions. While the motivations are different, both scientific endeavors involve understanding what happens to nuclei in intense neutron flux environments.

\section{Stockpile Stewardship}

In order to obtain information about the neutron flux during a nuclear weapons test, certain isotopes were used as flux monitors. The intense neutron flux of the test would initiate nuclear reactions on these monitors, creating radioactive nuclei that could be collected and measured after the test. Given the detection of these produced nuclei, it is possible to infer information about the neutron flux of the event, provided accurate cross section information is known not only for the loaded stable isotope, but for several nearby radioactive isotopes as well. Some of the nuclear reactions that take place in these tests are $(n, 2 n),(n, \gamma),\left(n, n^{\prime}\right),(n, p),(n, \alpha)$, and $(n, n p)$, with $(n, 2 n)$ and $(n, \gamma)$ being in general the more important 
reactions. But given the difficulty in performing neutron cross-section measurement on radioactive targets, most of the cross-sections used are determined from theory alone. Thus, a major effort in reducing the uncertainty of simulations of nuclear explosions is to reduce the uncertainty in the nuclear data used as input for the models. Table 1 below lists some of the reactions important to stockpile stewardship, the neutron energy range of interest, their relative importance and the desired accuracy. For a more detail description of the connection between stockpile stewardship and RIA, see reference [1].

TABLE 1. Neutron Reactions Important to Stockpile Stewardship

\begin{tabular}{|c|c|c|c|}
\hline Reaction & $\begin{array}{c}\text { Energy } \\
\text { Range } \\
(\mathbf{M e V})\end{array}$ & Importance & $\begin{array}{c}\text { Desired } \\
\text { Accuracy }\end{array}$ \\
\hline$(\mathrm{n}, \gamma)$ & $0.01-.0 .2$ & High & $10 \%$ \\
\hline$(\mathrm{n}, \mathrm{n})$ & $1-10$ & Low & $10 \%$ \\
\hline$(\mathrm{n}, 2 \mathrm{n})$ & $10-16$ & High & $3-5 \%$ \\
\hline$(\mathrm{n}, \alpha),(\mathrm{n}, \mathrm{pxn})$ & $0.1-16$ & Medium & $10 \%$ \\
\hline$(\mathrm{n}, \mathrm{f})$ & $0.1-16$ & High & $1-2 \%$ \\
\hline
\end{tabular}

\section{Astrophysics}

Stars with mass less than about 8 solar mass units will enter a stellar phase during which high neutron fluxes exist in the outer shell of the star. This enables a nucleosynthesis process known as the s-process, or slow neutron capture. In the s-process, seed nuclei experience successive neutron captures until an unstable isotope is reached and undergoes beta-decay forming the next heavier element. The process is repeated many times to form the elements between iron and bismuth. In most cases the nuclei either live long or short compared to the mean time between neutron capture events making determination of the next step of the process simple, either a neutron capture or beta decay, respectively. When these times are comparable, then detailed understanding of the neutron capture cross-section is required in order to understand in detail the effect the s-process has on the observed abundances of the various elements. Thus, one of the major goals of nuclear astrophysics is to precisely measure the neutron capture cross-sections for these branch point nuclei for neutron energies from 5-100 keV.

\section{HARVESTING ISOTOPES AT RIA}

Given the production limits at RIA, it should be possible to collect $10 \mu \mathrm{g}$ of many isotopes with a half- life as short as one day. Figure 1 below shows the generic relationship between required production rates and half-life for various collected amounts of an isotope with a mass number of 150 . The diamonds on the figure refer to some of the isotopes relevant to stockpile stewardship and their expected RIA production rate from the ANL RIA website [2]. The rates for low energy beams were used, i.e isotopes produced from the ISOL targets or fragmentation with a gas stopping cell. In some cases, the fragmentation harvesting limit may actually be higher than the one listed for low energy beams since it may not be necessary to impose the same operating conditions when harvesting is the goal. More details about harvesting can be found in reference [3].

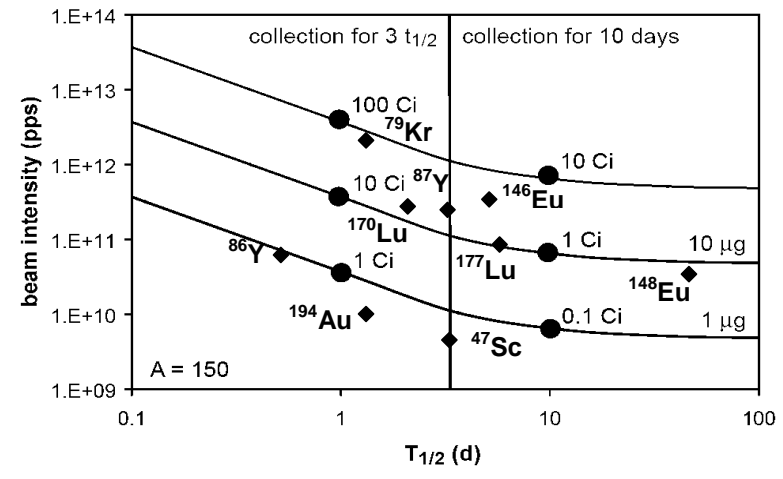

FIGURE 1. Plot showing required production rate versus half-life for collected various amounts of an isotope with a mass number of 150 . A collection time of three halflives or 10 days is assumed, whichever is shorter. The diamonds reflect the expected production rates at RIA [2] for various isotopes important to stockpile stewardship.

\section{PERFORMING THE MEASUREMENTS}

Given the variety of neutron reactions that need to be measured, a variety of neutron cross-section measurements techniques will need to be used. In many cases a simple activation measurement can be used, while in other cases, a more sophisticated reaction dependent prompt technique is needed.

\section{Activation}

The activation technique simply involves putting a sample in an intense neutron flux for some period of time and measuring the decay radiation signature of the reaction products. This technique is complicated for radioactive targets by the limited lifetime of the target material and the background radiation of the 
target itself. In most cases the activation product will only be one part in $10^{9}$ of the entire sample, so target purity is also a critical issue.

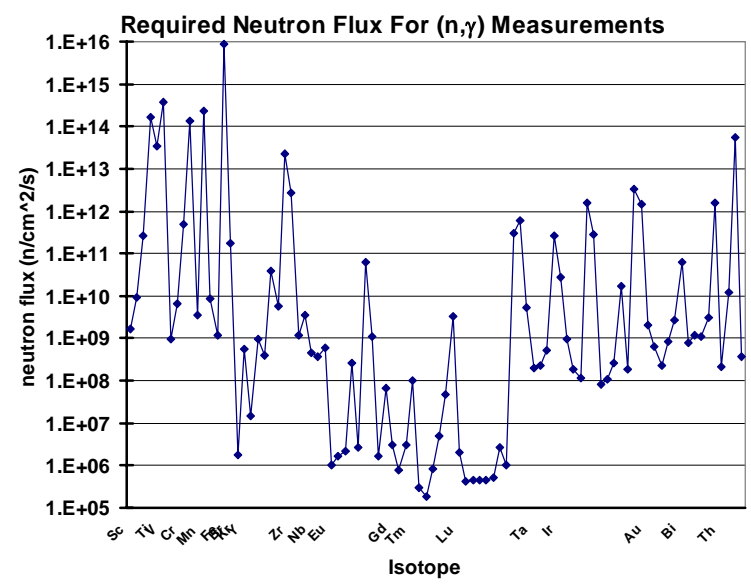

FIGURE 2. Plot of required neutron flux for performing activation measurements of $(n, \gamma)$ cross-section for various isotopes important to stockpile stewardship.

Figure 2 shows the required neutron flux for doing $(n, \gamma)$ activation measurements for isotopes important to stockpile stewardship. It is assumed that one thousand detected events are required, i.e. 3\% statistical error, and a $1 \%$ efficiency for detecting decaying activation products. The RIA production rates for the target material are used [2], and a 10 day collection period and a 10 day neutron irradiation are assumed. Also, the present estimate of the $(n, \gamma)$ crosssection at $40 \mathrm{keV}$ is used in the calculation. It should be noted that not all of these cross-sections listed in the figure can be measured via the activation technique. In some cases the reaction product is stable resulting in no residual radiation for detection. In some cases, it will be difficult to measure the signal given the large background of the target. It should also be pointed that the assumptions given above may not be appropriate for any given individual reaction which could result in a factor 10 reduction or increase in the require neutron flux.

\section{Prompt Measurements}

For those cases where activation measurement are impractical, a prompt technique, which measures each reaction as it occurs, is needed. For reactions which release a charged particle, detecting that charged particle offer a method of detecting the reactions. A magnetic or electric dipole of some type will probably be required to steer charged particles out of the neutron beam. This should also allow the particle detector to be shielded from the gamma irradiation coming from the target.

A much more difficult problem is to develop a prompt method for detecting $(n, 2 n)$ reactions. Frehaut and others [4] used a prompt technique to measure $(n, 2 n)$ reactions on stable target and some actinide targets. It is unclear whether that technique, which used a Gadolinium dopes scintillator to detect neutrons via their capture reactions, is viable for radioactive targets. The large gamma background, the small amount of target material, and the much higher required neutron flux present new challenges. The detector for this technique will most probably be a number of neutron detectors covering nearly $4 \pi$, since high overall efficiency is required. A highly segmented detector array is probably necessary as well to help mitigate the impact of gamma irradiation from the target. Much more work on this idea is required in order to develop a workable solution.

By far the most developed concept for prompt measurements is for the $(n, \gamma)$ reactions [5]. The technique would use an array of $\mathrm{BaF}_{2}$ detectors, like the DANCE detector, to do total gamma ray calorimetry to detect the neutron captures. A $1.9 \mathrm{MeV}$ proton beam is directed toward a lithium target to create low energy (5-100 keV) neutrons, with the target sample located roughly $4 \mathrm{~cm}$ away from the lithium production target. Given the low energy of the neutrons, time of flight could be used to measure the energy of the neutron as it takes $10 \mathrm{~ns}$ for the first neutron to hit the target and $40 \mathrm{~ns}$ for the first neutron to hit the $\mathrm{BaF}_{2}$ array, causing a much larger background signal. A pulsed beam structure is required for these beam experiments as it is necessary to wait for the rate of background $(n, \gamma)$ events to decay before the next burst of protons is put on targets. Repetition rates of $10 \mathrm{kHz}$ are ideal, but higher rates can be made to work with a $\mathrm{LiH}$ absorber ball inside the array. It should be noted that the required rates are reduced by a factor of 10 than those shown in figure 2 . The $\mathrm{BaF}_{2}$ array is nearly $100 \%$ efficient for detecting $(\mathrm{n}, \gamma)$ events, but 10 times more statistics is required to allow for the needed background subtraction.

\section{THE FACILITY}

Figure 3 illustrates the current conceptual design of the neutron source facility required at RIA to perform neutron cross section measurements on radioactive targets. Because of the required neutron energy range and variety of neutron reactions that need to be measured, several different experimental areas or 
neutron caves are included in the design. The distinguishing characteristic between the low and high energy cave is that the charged particle beam does not stop in the neutron production target for the high energy cave. The low energy cave would have a ${ }^{7} \mathrm{Li}(\mathrm{p}, \mathrm{n})$ beam line and a d(d,n) beam line and would provide neutrons up to about $12 \mathrm{MeV}$. The high energy cave would only have a deuteron break-up beam line and provide neutron with energies from 12$20 \mathrm{MeV}$. There is also a cave dedicated to prompt $(n, \gamma)$ measurements with a DANCE-like array as described above.

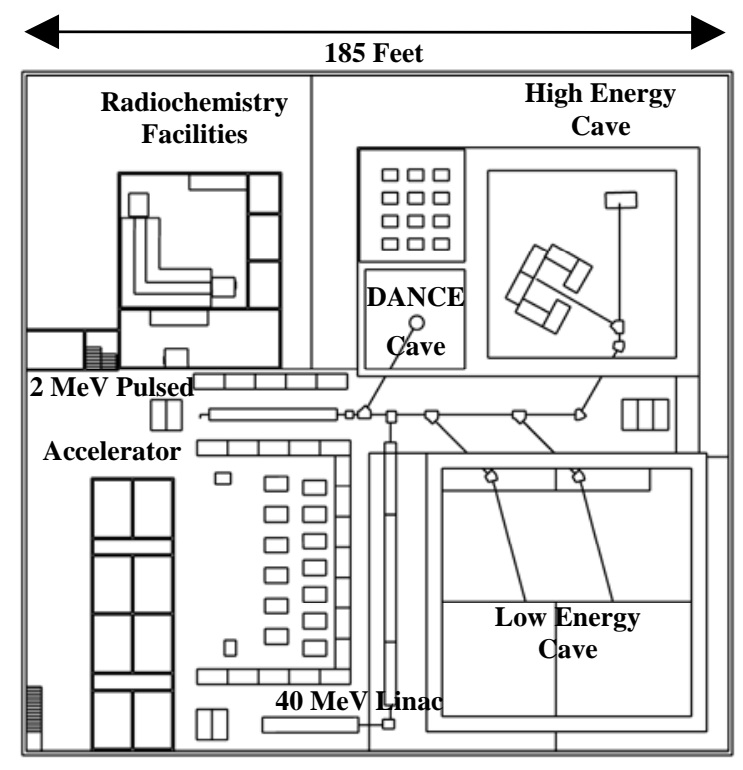

Figure 3. Diagram of conceptual design for neutron source with radiochemistry capabilities for doing neutron cross section measurements on radioactive target up from $10 \mathrm{keV}$ to $20 \mathrm{MeV}$ in neutron energy.

Also shown in figure 3 is the radiochemistry area. This area has general chemistry capability and consists hot cells capable for performing radiochemistry with 100 Curie samples of hard gamma radiation. Also present in this area is a mass separator to improve the purity of samples collected at RIA. This is particularly important for samples collected from the fragmentation line, where the purity will be lost by the stopping of the high energy fragments.

Table 2 lists the different accelerator options that have been explored. Two different accelerators are required, one that produces high average current and high energy for the activation measurements and high energy neutron production. The other accelerator only needs to produce $2 \mathrm{MeV}$ beams but must produce a high peak current consists with the timing structure required for prompt measurements. In both cases, there are several options each with advantages and disadvantages. Further study is needed before a final technology choice can be made.

\begin{tabular}{|c|c|c|}
\hline & Pros & cons \\
\hline \multicolumn{3}{|c|}{ HIGH AVERAGE CURRENT OPTIONS } \\
\hline $\begin{array}{l}\text { SCRF heavy-ion } \\
\text { linac at 57-175 } \\
\mathrm{MHz}\end{array}$ & $\begin{array}{l}\text { reliable, proven, } \\
\text { energy variable, } \\
\text { efficient }\end{array}$ & $\begin{array}{l}\text { cryogenic, } \\
\text { complex }\end{array}$ \\
\hline $\begin{array}{l}\text { NC-RF linac at } \\
425 \mathrm{MHz}\end{array}$ & reliable, proven & $\begin{array}{l}\text { need high beam } \\
\text { loading for } \\
\text { efficiency }\end{array}$ \\
\hline Pelletron & $\begin{array}{l}\text { good energy } \\
\text { agility, DC beam }\end{array}$ & low current, size \\
\hline $\begin{array}{l}\text { Cyclotron at } 25 \\
\mathrm{MHz}\end{array}$ & $\begin{array}{l}\text { provides } 1 \mathrm{nsec} \\
\text { pulses at } 100 \mathrm{nsec}\end{array}$ & $\begin{array}{l}\text { low current, } \\
\text { limited energy } \\
\text { agility, big }\end{array}$ \\
\hline \multicolumn{3}{|c|}{ HIGH PEAK CURRENT OPTIONS } \\
\hline Induction linac & $\begin{array}{l}\text { potential for very } \\
\text { high peak current }\end{array}$ & untried for ions \\
\hline $\begin{array}{l}\text { Rapid cycling } \\
\text { synchrotron } \\
\text { (RCS) } \\
\end{array}$ & $\begin{array}{l}\text { could provide } \\
\text { ideal beam pulse } \\
\text { structure }\end{array}$ & $\begin{array}{l}\text { underdeveloped, } \\
\text { large size }\end{array}$ \\
\hline Dynamitron & $\begin{array}{l}\text { good energy } \\
\text { agility, AC CW } \\
\text { beam }\end{array}$ & $\begin{array}{l}\text { low current, } \\
\text { unproven for ions }\end{array}$ \\
\hline
\end{tabular}

\section{CONCLUSION}

RIA enables new opportunities in neutron crosssection measurements on unstable nuclei. However, performing these measurements require certain capabilities be added to the RIA site. We are working hard with the RIA community to insure these measurements can and will take place. This work was performed under the auspices of the U.S. Department of Energy by the University of California, Lawrence Livermore National Laboratory under contract No. W7405-Eng-48 and by Los Alamos National Laboratory under contract No. W-7045-ENG-36.

\section{REFERENCES}

1. Ahle, L. et. al., "Science Based Stockpile Stewardship and RIA" in Accelerator Applications in a Nuclear Renaissance, Proceedings of Sixth International Meeting on Nuclear Applications of Accelerator Technology, American Nuclear Society, June 2003, pp. 286-291.

2. http://www.phy.anl.gov/ria/ria_yields/yields_home.html.

3. Hausmann, M., et. al., "Collection of separated isotopes for radioactive target production at RIA", Proceedings of Radioactive Nuclear Beams 6, September 2003.

4. Frehaut, J., Nucl. Inst. And Meth., 135, 511-518 (1976).

5. R. Reifarth, et al., Nucl. Inst. And Meth., A 524, 215-226 (2004). 